\title{
Neurofeedback for attention-deficit/hyperactivity disorder: meta-analysis of clinical and neuropsychological outcomes from randomized controlled trials
}

Running title: Neurofeedback for ADHD

Samuele Cortese, M.D., Ph.D.*, Maite Ferrin, M.D, Ph.D.*, Daniel Brandeis, Ph.D., Martin

Holtmann, M.D., Pascal Aggensteiner, M.A., David Daley, Ph.D., Paramala Santosh, M.D., Ph.D., Emily Simonoff, M.D., Ph.D., Jim Stevenson, Ph.D., Argyris Stringaris, M.D., Ph.D., MRCPsych., \& Edmund J.S. Sonuga-Barke, Ph.D. on behalf of the European ADHD Guidelines Group (EAGG). *Joint first authors.

Samuele Cortese, Maite Ferrin, Jim Stevenson and Edmund J.S. Sonuga-Barke are with the Academic Unit of Psychology, Developmental Brain-Behaviour Laboratory, University of Southampton, UK; Samuele Cortese is also with the New York University Child Study Center, NY; Maite Ferrin is also with the Huntercombe Hospital Maidenhead, UK; Edmund J.S. Sonuga-Barke is also with the Department of Experimental Clinical and Health Psychology, Ghent University, Belgium and the Aarhus University, Denmark; Daniel Brandeis and Pascal Aggensteiner are with the Department of Child and Adolescent Psychiatry and Psychotherapy, Central Institute of Mental Health, Medical Faculty Mannheim, Heidelberg University, Mannheim, Germany; Daniel Brandeis is also with the Department of Child and Adolescent Psychiatry, University of Zurich, Switzerland, the Center for Integrative Human Physiology, University of Zurich, Switzerland, and the Neuroscience Center Zurich, University of Zurich and ETH Zurich, Switzerland; Martin Holtmann is with the LWL-University Hospital for Child and Adolescent Psychiatry, Ruhr University Bochum, Germany; David Daley is with the School of Medicine \& MindTech Institute of Mental Health, University of Nottingham, UK; Paramala Santosh, Emily Simonoff, and Argyris Stringaris are with the Institute of Psychiatry, Psychology and Neurosciences, King's College London, UK and with the Maudsley Hospital, London, UK. 
EAGG members: Asherson Phil, Banaschewski Tobias, Brandeis Daniel, Buitelaar Jan, Coghill

David, Cortese Samuele, Daley David, Danckaerts Marina, Dittmann Ralf W, Dopfner Manfred, Ferrin Maite, Hollis Chris, Holtmann Martin, Konofal Eric, Lecendreux Michel, Rothenberger Aribert, Santosh Paramala, Sergeant Joseph A, Simonoff Emily, Sonuga-Barke Edmund J, Soutullo Cesar, Steinhausen Hans-Christoph, Stevenson Jim, Stringaris Argyris, Taylor Eric, van der Oord Saskia, Wong Ian, Zuddas Alessandro.

Corresponding Author: Edmund J.S. Sonuga-Barke, Ph.D., Developmental Brain-Behaviour Laboratory, Psychology Academic Unit, University of Southampton, UK. Tel: +442380594604 ; Fax: +44238059500; E-mail: ejb3@ soton.ac.uk

Abstract word count: 214

Text word: 6996

Tables: 2

Figures: 3

Supplemental texts: 2; Supplemental Tables: 1; Supplemental Figures: 6

Acknowledgments: Support for meetings was received from Brain Products GMBH, JanssenCilag, Lilly, Medice, Shire, and Vifor. No honoraria were received, and funders made no input into the review process/writing of the paper. The authors thank Eugene Arnold, M.D., Ohio State University and Hanna Christiansen, Ph.D., Marburg University, Germany for providing additional information on studies 


\begin{abstract}
Objective: We performed meta-analyses of randomized controlled trials to examine the effects of neurofeedback on attention-deficit/hyperactivity disorder (ADHD) symptoms and neuropsychological deficits in children/adolescents with ADHD. Method: We searched Pubmed, Ovid, Web of Science, ERIC and CINAHAL through August 30, 2015. Random-effects models were employed. Studies were evaluated with the Cochrane Risk of Bias (RoB) tool. Results: We included 13 trials (520 ADHD participants). Significant effects were found on ADHD symptoms rated by assessors most proximal to the treatment setting, i.e., the least blinded outcome measure (Standardized Mean Difference [SMD]: ADHD total symptoms=0.35, 95\% CI=0.11-0.59; inattention $=0.36,95 \% \mathrm{CI}=0.09-0.63$; hyperactivity/impulsivity $=0.26,95 \% \mathrm{CI}=0.08-0.43)$. Effects were not significant when probably blinded ratings were the outcome or in trials with active/sham controls. Results were similar when only Frequency Band Training trials, the most common neurofeedback approach, were analysed separately. Effects on laboratory measures of inhibition $(\mathrm{SMD}=0.30,95 \% \mathrm{CI}=-0.10-0.70)$ and attention $(\mathrm{SMD}=0.13,95 \% \mathrm{CI}=-0.09-0.36)$ were not significant. Only four studies directly assessed if learning occurred after neurofeedback training. The risk of bias was unclear for many RoB domains in most studies. Conclusions: Evidence from well-controlled trials with probably blinded outcomes currently fails to support neurofeedback as an effective treatment for ADHD. Future efforts should focus on implementing standard neurofeedback protocols, ensuring learning and optimizing clinically relevant transfer.
\end{abstract}




\section{Introduction}

Attention-deficit/hyperactivity disorder (ADHD) is a common neurodevelopmental disorder characterized by age-inappropriate and impairing inattention and/or hyperactivity/impulsivity ${ }^{1,2}$. Among currently available treatment options, psychostimulant and non-stimulant medications are efficacious, at least in the short-term, and widely used ${ }^{3}$. Non-pharmacological interventions - both dietary and psychological - have been also extensively investigated ${ }^{4-7}$. Among nonpharmacological approaches, neurofeedback has been considered a promising ADHD treatment strategy since the early $1970 \mathrm{~s}^{8-10}$. When applied to ADHD, neurofeedback is intended to reduce ADHD symptoms by targeting aberrant patterns of brain activity thought to underpin the condition. Neurofeedback is implemented through the training of self-regulation using operant reinforcement procedures; learning of self-regulation is thus a key mechanism. To achieve this aim, electroencephalogram (EEG) indices of interest are converted into visual or acoustic signals and fed back automatically in real time to the patient. For instance, cortical activity may be represented by the height or speed of a ball, plane or cartoon character presented using animation on a computer screen. In this case, learning occurs when the object rises, falls, or advances more quickly in response to patients' regulated changes in brain activity. Two general neurofeedback approaches have been used to treat ADHD: Frequency Band Training (FBT) and Slow Cortical Potential training (SCP). When applied to ADHD, the former is intended to target alterations of cortical electrical oscillations thought to be associated with ADHD, namely elevations of slow, relative to fast, brainwave activity - especially in the frontal lobes (e.g., theta versus beta frequency ${ }^{11}$ ). The latter aims to regulate cortical excitation thresholds by focusing on activity generated by external cues (similar to event-related potentials), focusing primarily on EEG components registered in the late latency range, i.e., several seconds after the cue. For instance, this form of training has been used to target the contingent negative variation $(\mathrm{CNV})$ which occurs during this time window and is involved in effective preparation, decision-making and time estimation, which have all been found to be deficient in individuals with ADHD, or at least in subgroups of them ${ }^{12,13}$. 
The efficacy of non-pharmacological treatments for ADHD, including neurofeedback, has been subject to a number of earlier meta-analytic reviews ${ }^{14-16}$. However, these have sometimes been difficult to interpret because of the inclusion of studies with weak experimental designs (e.g., no control arm, non-random allocation or the use of unblinded measures), as discussed in SonugaBarke et al. ${ }^{17}$. On behalf of the European ADHD Guidelines Group (EAGG), Sonuga-Barke et al. ${ }^{17}$ attempted to address these limitations through a meta-analysis of non-pharmacological interventions which included only randomised controlled trials (RCTs). It also addressed the issue of blinding by comparing outcomes rated by individuals judged to be most proximal to the therapeutic setting (often parents poorly blinded and invested in the therapeutic outcome) and those provided by reporters judged to be probably blinded. They found that the effects of neurofeedback on ADHD total symptoms based on most proximal ratings were highly significant $(\mathrm{SMD}=0.59,95 \% \mathrm{CI}=0.31$ 0.87). However, when only probably blinded measures were employed, the effects became nonsignificant $(\mathrm{SMD}=0.29 ; 95 \% \mathrm{CI}=-0.02-0.61)$. More recently, Micolaud-Franchi and colleagues ${ }^{18}$ followed a similar approach, focusing their analyses on ADHD core symptoms, but with a smaller set of studies $(n=5)$ limited to trials with particular control conditions. As in Sonuga-Barke et al. ${ }^{17}$, they found a significant, positive effect of neurofeedback on ADHD core symptoms when considering most proximal raters. Probably blinded scores were attenuated and were significant only for symptoms of inattention.

Applying the same meta-analyses protocol used in recent EAGG reviews of behavioural interventions ${ }^{5}$ and cognitive training ${ }^{4}$, we here extend the focus of meta-analytic evidence relating to neurofeedback for ADHD in a number of ways. First, we included, among the outcomes, not only specific ADHD behavioral dimensions (i.e., inattention and impulsivity/hyperactivity) but also ADHD-related neuropsychological deficits such as inhibitory dysfunction. The latter may be important as they may take us closer to neural mediators of the behavioural effects of neurofeedback ${ }^{9}$. Second, we addressed the relative efficacy of different types of neurofeedback by restricting sub-analyses to specific types of treatment protocols, i.e., FBT. Third, we examined the 
impact of different aspects of trial design (e.g., use of a sham/placebo design) or pragmatic "dosage" characteristics of neurofeedback implementation (i.e., number of sessions). Fourth, we addressed the crucial question of whether neurofeedback-related learning at the neural level was investigated and/or demonstrated in available trials ${ }^{9}$. Fifth, we examined whether the neurofeedback protocols employed in these studies could be considered "standard" in terms of the criteria discussed by Arns et al. ${ }^{19}$, which include elements related to EEG bands/measures, electrode placement and type and feedback following learning. Finally, we applied, for the first time in a meta-analysis of neurofeedback for ADHD, a rigorous assessment of study bias, i.e., the Cochrane risk of bias tool $(\mathrm{RoB})^{20}$.

\section{Method}

The EAGG protocol was originally registered on the International Prospective Register of Systematic Reviews PROSPERO (http://www.crd.york.ac.uk/PROSPERO, protocol number: CRD42011001393). As in ${ }^{5}$ and ${ }^{4}$, the original protocol was adapted to take account of the broader scope of this systematic review/meta-analysis. Most crucially, given that the scope of this analysis included neuropsychological measures, the mandatory requirement for studies to have ADHD symptoms-related outcome no longer applied (i.e., we included also studies presenting only neuropsychological outcomes).

\section{Inclusion and Exclusion Criteria}

To ensure high levels of methodological adequacy as recommended by the Cochrane group and to avoid the inevitable bias caused by dependence on investigators agreeing to provide data from unpublished studies ${ }^{20}$, only published studies were included. Only RCTs using neurofeedback training were retained. Participants in the trials were required to be between 3 and 18 years of age, and to have a diagnosis of ADHD diagnosis (any subtype) or Hyperkinetic Disorder (HKD), or meet accepted cut-offs on validated ADHD symptom rating scales. Trials that selected children with ADHD who had rare comorbid disorders (e.g., fragile X syndrome) were excluded. Control 
conditions allowed were "treatment as usual," "wait list," "active" or "placebo/sham" (i.e., involving other forms of alternative training regimen). As per the EAGG protocol, trials where neurofeedback was compared only with optimised medication or where the additional effects of neurofeedback when added to optimized medication were excluded. Trials where medication was part of background normal clinical provision in either the control or the active arm were included.

\section{Search Strategy}

Details about the search strategy/syntax for each database are reported in Supplemental Text 1, available online. The final search was updated on August 30, 2015. Independent searches were conducted by Samuele Cortese and Maite Ferrin, leading to the same number of references.

\section{Outcome Measures}

To provide analytical robustness and in line with previous EAGG meta-analyses 4, 5, 17, 20 , analyses of outcome domains were only considered reliable if at least five RCTs were available. The planned outcomes included: ADHD symptoms (total ADHD and inattention and hyperactivity/impulsivity symptoms separately), neuropsychological laboratory-based measures, measures of academic functioning, and rating of severity of symptoms of comorbid conditions (e.g., oppositional defiant disorder or anxiety disorders).

\section{Study Selection}

Retrieved references were independently screened and blindly double-coded for eligibility by Samuele Cortese and Maite Ferrin. Any disagreement was resolved by a senior author (Edmund Sonuga-Barke).

\section{Study bias assessment}

Study quality was assessed independently by pairs of raters from the authorship group using the Cochrane $\operatorname{RoB}^{20}$. RoB domains included: selection bias, performance bias, detection bias, attrition bias, and other bias. Any disagreement was resolved through consensus.

\section{Data Extraction and Statistical Analysis}


Trial information was entered into RevMan $5.3^{21}$. Data extraction was independently performed and cross-checked by the first two authors. SMD was calculated as mean pre- to posttreatment change in the intervention group minus the mean pre- to post treatment change in the control group, divided by the pooled pre-test standard deviation with a bias adjustment ${ }^{22}$. SMDs for each trial were combined using the inverse variance method. Given the inherent heterogeneity of studies, random effects models were used. The $\mathrm{I}^{2}$ statistic was calculated to estimate between-trial SMD heterogeneity. When multiple measurements were available for an outcome, the most frequently reported outcome across trials or the outcome that was judged to better tap the core of the construct was selected. To be consistent with the EAGG protocol, we considered as probably blinded those outcomes rated by an individual who was likely unaware of treatment allocation. Most proximal ratings were based on assessors close to the therapeutic setting and often unblinded. These ratings typically constituted a trial's own primary outcome measure and were therefore the assessment most available for analysis.

Selection of most proximal and probably blinded ratings was based on independent judgements and consensus of three authors (Samuele Cortese, Maite Ferrin, Edmund SonugaBarke). Where two or more probably blinded ratings were available (which was sometimes the case on sham controlled trials) what was deemed the best probably blinded outcome was selected for analysis, as in Cortese et al. ${ }^{4}$. When there were two or more neuropsychological outcome measures, the one most frequently reported across the relevant trials was selected. Four sensitivity analyses were conducted. The first included only trials employing an active or sham control. The second was restricted to FBT trials. The third examined the effect of co-treatment with medication and was restricted to studies with no/low levels of medication ( $<30 \%$ of participants on medication, as per EAGG protocol). The final sensitivity analysis included only studies meeting the criteria defining a standard neurofeedback methodology described by Arns et al. ${ }^{19}$ (see Supplemental Text 2, available online). Meta-regression was conducted to assess the effects of number of training sessions. Publication bias was assessed with funnel plots and Egger's tests. Analyses were 
conducted using RevMan $5.3^{21}$ and STATA $13.1^{23}$.

\section{Results}

Thirteen trials ${ }^{24-36}$ met entry criteria. Figure 1 presents the PRISMA selection flowchart. Supplemental Table S1, available online, provides a list of excluded papers with reasons for exclusion. Retained studies included a total of 520 ADHD subjects. Table 1 gives information about the characteristics of the retained trials. Results of all analyses are summarized in Table 2. There were less than five studies available to examine some of the planned outcomes such as working memory, parent ratings of executive functioning (e.g., Behaviour Rating Inventory of Executive Function ${ }^{37}$ ), academic functioning (e.g., reading or arithmetic ability), or comorbid conditions (e.g., ODD, anxiety). Therefore, we did not perform analyses for such outcomes.

When most proximal assessments were the outcome, there was a small-to-moderate (SMD < 0.5) but significant effect on inattention, impulsivity/hyperactivity and total ADHD symptoms (Figure 2). In sensitivity analyses considering only trials with an active/sham control, the effects dropped to non-statistically significant levels for total ADHD and inattention symptoms. The effect was significant for hyperactivity/impulsivity but with a small effect size (SMD=0.25) and the 95\% CI was wide ( $\mathrm{CI}=0.03-0.47)$. When probably blinded outcomes were analysed, effects sizes for ADHD outcomes dropped further and none were significant (Figure 3). Results were also not significant when considering only probably blinded measures from trials with active/sham control (Table 2). When considering only trials with FBT, results were significant for ADHD total $(\mathrm{SMD}=0.37,95 \% \mathrm{CI}=0.09-0.64)$ and hyperactive/impulsive symptoms $(\mathrm{SMD}=0.26,95 \% \mathrm{CI}=0.06-$ 0.46) from most proximal raters, but not for ADHD inattentive symptoms or any ADHD symptoms rated by probably blinded assessors. There were insufficient trials $\left(n=2^{30,32}\right)$ for an analysis focused on SCP training (two studies also used both SCP and FBT ${ }^{31,33}$ ). When pooling only trials with no/low medication, results were significant only for ADHD inattentive symptoms, most proximal $(\mathrm{SMD}=0.59,95 \% \mathrm{CI}=0.31-0.88)$ and hyperactive/impulsive symptoms, most proximal $(\mathrm{SMD}=0.32$, $95 \% \mathrm{CI}=0.06-0.58)$. 
When the analysis was restricted to only the seven trials that met Arns et al. ${ }^{19}$ criteria for use of standard protocol, the SMDs increased for most proximal outcomes although the $95 \%$ CIs were wide (Table 2). There were only three trials ${ }^{27,30,31}$ that both employed a standard protocol and had probably blinded measures. In an exploratory analysis with these three trials, the effect on total ADHD symptoms was significant $(\mathrm{SMD}=0.36)$, although the $95 \% \mathrm{CI}$ was large (0.04-0.69).

The analysis of neuropsychological outcomes required the pooling of diverse neuropsychological measures within general domains (Table 1). Effects on laboratory measures of inhibition $(\mathrm{SMD}=0.30,95 \% \mathrm{CI}=-0.10-0.70)$, and attention $(\mathrm{SMD}=0.13,95 \% \mathrm{CI}=-0.09-0.36)$ were not significant.

Direct evidence that neurofeedback training led to learning was gathered in only four trials $27,29,33,36$ and was positive for just one ${ }^{27}$. In one study ${ }^{29}$ there was a partial learning effect. In Heinrich et al. ${ }^{32}$ there was indirect evidence of learning: the increase of the contingent negative variation (a slow cortical potential) suggests that children learned what was trained.

Studies varied considerably in terms of risk of bias (Supplemental Figures S1 and S2, available online). For approximately half of the RoB domains across studies, the level of risk was unclear. For those categories that could be determined, eight trials were rated as having a high risk of bias in at least one domain. In general, the major concern (i.e., high risk of bias) related to blinding of participants, personnel, and assessors. Among "other bias", funding was not clearly stated in a sizable portion of studies.

Funnel plots and Egger test results (Supplemental Figures S3-S4, available online) suggested little evidence of publication bias although the number of trials was insufficient to establish a reliable estimate. Finally, meta-regression analyses did not support a relationship between number of training sessions and most proximal or probably blinded outcomes (Supplemental Figures S5 and S6, available online).

\section{Discussion}


The rationale for the use of neurofeedback for ADHD rests on the idea that promoting normalization or self-regulation of brain activity will translate into improved cognitive and behavioural control that is deficient in individuals ADHD. A previous meta-analysis ${ }^{17}$, based on a limited number of trials $(n=8)$, was inconclusive with regard to the efficacy of neurofeedback for ADHD symptoms. Although there were moderate but highly significant effects (SMD $=0.59 ; 95 \%$ $\mathrm{CI}=0.31-0.87$ ) for ADHD outcomes rated by most proximal assessors, these were not significant when only probably blinded outcomes were considered (SMD $=0.29 ; 95 \%$ CI: $-0.02-0.61)$. The current meta-analysis, including an additional five RCTs, provides what is likely to be a more reliable estimate of the effects of neurofeedback for ADHD. Importantly, the additional statistical power also allowed us to explore the impact of neurofeedback type, the effect of type of control, the impact of number of NF sessions, and the value of using standard neurofeedback procedures. Although it does not provide a definitive statement as to the value of neurofeedback, the current analysis has clarified a number of issues.

In general, the effect size estimates in the current analysis are substantially smaller compared to the previous one by Sonuga-Barke et al. ${ }^{17}$. This is due to the smaller effect sizes reported in the most recent trials, not included in Sonuga-Barke et al. ${ }^{17}$. For instance, SMDs dropped for most proximal and probably blinded total ADHD outcomes by $41 \%$ and $49 \%$, respectively - although the analysis based on most proximal ratings of ADHD core symptoms remained significant. Crucially, as in the previous meta-analysis ${ }^{17}$, when the risk of biased effect size estimates was reduced either by selecting probably blinded outcomes or limiting analysis to trials with a high-quality control arm (sham or active), effects were no longer significant. Importantly, and in contrast to previous meta-analyses ${ }^{15,18}$, there was no evidence for a particular benefit with regard to inattention symptoms - which have previously been hypothesized to be more amenable to neurofeedback. This was also reflected in our failure to find effects on laboratory measures of attention such as the continuous performance test. 
Previously, Sonuga-Barke et al. ${ }^{17}$ have argued that the substantial drop in SMDs between most proximal and probably blinded analyses is likely to be result of biases in perception in favour of the active treatment when one relies on observations by raters aware of treatment allocation. However, there are some other explanations. For instance, it is possible that probably blinded ratings are, for some reason, less sensitive to change than most proximal measures - perhaps either because of the instruments used or the person rating. Teachers, for instance, may be less sensitive to change than parents. However, essentially the same questionnaires were completed by both these types of raters. It is also possible that proximal ratings accurately reflected real improvements in the setting where the treatment was delivered but these effects failed to generalise to more distal settings where blinded measures were recorded. This explanation seems unlikely as probably blinded measures were collected in the treatment setting also for some trials and these followed the same pattern. The type of neurofeedback protocol implemented in the trials did not seem to be an important factor in determining the results. When we restricted the analysis to trials using some form of FBT such as that focusing on alteration of the ratio between slow theta and faster beta oscillations, there was no increase in the effects of treatment. There was an insufficient number of trials $(n=2)^{30,32}$ using SCP training exclusively to state any firm assertion about this approach. The results raise the question of whether current training protocols have the appropriate treatment target. For instance, the developmental stability of EEG frequency band alterations in ADHD from childhood into adulthood have been questioned ${ }^{11,38,39}$, while the most commonly observed ADHD effects with regard to evoked brain responses relate to early rather than later components not targeted in current neurofeedback protocols. Therefore, the rationale for theta-beta feedback have been very critically discussed (e.g., ${ }^{9,40}$ ).

The value of a treatment meta-analysis is of course constrained by the methodology of the trials they include. It is, therefore, possible that the results of our meta-analysis reflect the methodological weaknesses of the included studies rather than the weakness of neurofeedback as such. Indeed, the current set of 13 trials, taken as whole, had a number of methodological short- 
comings. First, only four studies tested ${ }^{27,29,33,36}$, and then just one ${ }^{27}$ reported directly and positively, whether neurofeedback training had actually led to learning as indexed by changes/improvement at the electrophysiological level. In the one positive trial ${ }^{27}$, the mediating role of changes in the electrophysiological signature leading to changes at symptom level was not investigated. This is a crucial point because if neurofeedback cannot bring about the expected changes at the neural level, then treatment effects are more likely to be artifacts of some other nonspecific aspect of the training. Such a situation may be similar to cognitive training targeting but not improving working memory, or to a drug with an established neurotransmitter profile not reaching the corresponding neural target system in the patient's brain. On the other hand, tests for learning of neural self-regulation, and addressing relations between learning and clinical improvement, would also need to consider more complex models and alternative mechanisms, for example allowing for initial, delayed, and nonlinear types of learning and translation ${ }^{41}$, before concluding that effects are non-specific. Further research should address whether possible neuronal modifications underpin putative behavioral changes in ADHD symptoms following neurofeedback.

A number of groups have defined what constitutes a standard neurofeedback protocol in terms of the number of training sessions, the reinforcement parameters operating, the EEG montage etc. (see Vernon et al. $2004^{42}$ for a justification). Interestingly only $54 \%$ of the studies in the current analysis met such a threshold. When we restricted the analysis to that subset of trials our results were somewhat mixed - the effect size for total ADHD and inattention increased by about 20\%; however, for all most proximal outcomes, $95 \%$ CIs were wide and close to non significance. Unfortunately, there were only three studies with a standard protocol including probably blinded ratings $27,30,31$, so that firm conclusions on the value of standardised protocols as defined by Arns et al. ${ }^{19}$ cannot be drawn. In addition, the level of methodological rigour specifically related to RCT conduct, as explored by the RoB, was in general unclear. The level of blinding was unclear or insufficient in many studies. Additionally, one particularly striking omission in the majority of trials was the report of possible potential conflicts of interest. This would seem to be a major oversight in 
the current literature, given the growing presence of neurofeedback training companies in the commercial treatment marketplace. Given these limitations, it seems that without evidence for the learning of self-regulation and given the wide spread use of non- standard neurofeedback protocols it is hard to draw definitive conclusions about the ultimate value of neurofeedback approaches.

EAGG have also recently completed meta-analyses for behavioural interventions and cognitive training using the same core protocol as used here ${ }^{4,5}$. There are striking similarities but also some differences between the results of the present meta-analysis and those from these recent EAGG meta-analyses. In terms of effects on ADHD symptoms, neurofeedback, cognitive training and behavioural interventions show almost identically sized positive and statically significant effects on total ADHD symptoms scores rated by most proximal assessors (SMD: behavioural interventions $=0.35$; cognitive training $=0.37$; neurofeedback $=0.35$ ). Furthermore, in all three cases, the effects drop substantially to non-significant levels when probably blinded outcomes are used. This is more evident for behavioural interventions where the effects drop to zero but is also substantial for neurofeedback and cognitive training ( $\mathrm{SMD}=0.15$ and 0.20 respectively). However, one quite striking difference between the three meta-analyses is that both behavioural interventions and cognitive training had predictable positive effects on outcomes other than ADHD. For instance, for working memory training, there were highly significant effects on neuropsychological measures of working memory, while behavioural interventions improved parenting rated by independent observers and had positive effects on probably blinded measures of conduct problems. By contrast, we did not find evidence for effects of neurofeedback on neuropsychological outcomes.

Caution is required when interpreting these findings given a number of limitations, in addition to the issues raised with regard to the nature of the trials above. First, effects size estimates may be inflated because of the failure to report intention to treatment analyses in most trials. Second, there were insufficient trials measuring important outcomes such as working memory task performance, academic skills, general functional impairment, IQ and other mental health problems such as conduct problems. Third, few trials included long-term outcomes (see Table 1) to allow an 
evaluation of the extent to which effects on clinical symptoms grew over time or effects on neuropsychological processes persisted. Fourth, no trial recruited subjects based on the presence of ADHD-related deficits in EEG signature (i.e., altered theta-beta ratio). This may limit the chance for neurofeedback related improvements in symptoms. Fifth, it was necessary to pool data from diverse measures from different tasks to have sufficient trials to analyse neuropsychological functions. Although in principle the pooled measures tapped the same neuropsychological domain, this inevitably was a somewhat arbitrary process which likely increased SMD heterogeneity. Additionally, ADHD is a pathophysiologically heterogeneous disorder and distinct EEG subtypes have been described (e.g., cortical hyperarousal $v s$. hypoarousal subtypes). Patients might require more specific and tailored training targeting different deficits associated with ADHD. Combining different neuroimaging approaches with NF training might be a useful approach in the future.

Finally, the range of number of sessions across studies may have been too restricted to allow the detection of possible effect of session number.

In summary, the current meta-analysis shows that evidence from well controlled and with probably blinded outcomes does not support neurofeedback as an effective treatment for ADHDeither in terms of ADHD symptoms or other cognitive correlates. Future research should focus on (i) identifying the most appropriate electrophysiological treatment target; (ii) increasing the use of standard EEG and learning protocols (iii) developing new methods to optimise the chances that neurofeedback leads to learning at the brain level and (iv) identifying predictors of treatment response for individual patients or at least in distinctive subgroups of children. 


\section{References}

1. Faraone S, Asherson P, Banaschewski T et al. Nature Reviews: Disease Primers [published online ahead of print August 2015], doi: 10.1038/nrdp.2015.20.

2. Polanczyk GV, Salum GA, Sugaya LS, Caye A, Rohde LA. Annual research review: A meta-analysis of the worldwide prevalence of mental disorders in children and adolescents. $\mathbf{J}$ Child Psychol Psychiatry 2015;56(3):345-365.

3. Taylor E. Uses and misuses of treatments for ADHD. The second Birgit Olsson lecture. Nord J Psychiatry 2014;68(4):236-242.

4. Cortese S, Ferrin M, Brandeis D et al. Cognitive training for attention-deficit/hyperactivity disorder: meta-analysis of clinical and neuropsychological outcomes from randomized controlled trials. J Am Acad Child Adolesc Psychiatry 2015;54(3):164-174.

5. Daley D, Van der Oord S, Ferrin M et al. Behavioral Interventions in AttentionDeficit/Hyperactivity Disorder: A Meta-Analysis of Randomized Controlled Trials Across Multiple Outcome Domains. J Am Acad Child Adolesc Psychiatry 2014;53(8):835-847.

6. Fabiano GA, Schatz NK, Aloe AM, Chacko A, Chronis-Tuscano A. A systematic review of meta-analyses of psychosocial treatment for attention-deficit/hyperactivity disorder. Clin Child Fam Psychol Rev 2015;18(1):77-97.

7. Stevenson J, Buitelaar J, Cortese S et al. Research Review: The role of diet in the treatment of attention-deficit/hyperactivity disorder - an appraisal of the evidence on efficacy and recommendations on the design of future studies. J Child Psychol Psychiatry 2014;55(5):416-27. 
8. Arns M, Heinrich H, Ros T, Rothenberger A, Strehl U. Editorial: Neurofeedback in ADHD. Front Hum Neurosci 2015;9:602.

9. Holtmann M, Sonuga-Barke E, Cortese S, Brandeis D. Neurofeedback for ADHD: a review of current evidence. Child Adolesc Psychiatr Clin N Am 2014;23(4):789-806.

10. Lubar JF, Shouse MN. EEG and behavioral changes in a hyperkinetic child concurrent with training of the sensorimotor rhythm (SMR): a preliminary report. Biofeedback Self Regul 1976;1(3):293-306.

11. Arns M, Conners CK, Kraemer HC. A decade of EEG Theta/Beta Ratio Research in ADHD: a meta-analysis. J Atten Disord 2013;17(5):374-383.

12. Holtmann M. Commentary: persistent time estimation deficits in ADHD? From developmental trajectories to individual targets for intervention - reflections on Doehnert et al. (2013). J Child Psychol Psychiatry 2013;54(3):271-272.

13. Doehnert M, Brandeis D, Schneider G, Drechsler R, Steinhausen HC. A neurophysiological marker of impaired preparation in an 11-year follow-up study of attentiondeficit/hyperactivity disorder (ADHD). J Child Psychol Psychiatry 2013;54(3):260-270.

14. Nigg JT, Lewis K, Edinger T, Falk M. Meta-analysis of attention-deficit/hyperactivity disorder or attention-deficit/hyperactivity disorder symptoms, restriction diet, and synthetic food color additives. J Am Acad Child Adolesc Psychiatry 2012;51(1):86-97.

15. Arns M, de RS, Strehl U, Breteler M, Coenen A. Efficacy of neurofeedback treatment in ADHD: the effects on inattention, impulsivity and hyperactivity: a meta-analysis. Clin EEG Neurosci 2009;40(3):180-189.

16. Fabiano GA, Pelham WE, Jr., Coles EK, Gnagy EM, Chronis-Tuscano A, O'Connor BC. A meta-analysis of behavioral treatments for attention-deficit/hyperactivity disorder. Clin Psychol Rev 2009;29(2):129-140. 
17. Sonuga-Barke EJ, Brandeis D, Cortese $\mathrm{S}$ et al. Nonpharmacological interventions for ADHD: systematic review and meta-analyses of randomized controlled trials of dietary and psychological treatments. Am J Psychiatry 2013;170(3):275-289.

18. Micoulaud-Franchi JA, Geoffroy PA, Fond G, Lopez R, Bioulac S, Philip P. EEG neurofeedback treatments in children with ADHD: an updated meta-analysis of randomized controlled trials. Front Hum Neurosci 2014;8:906.

19. Arns M, Heinrich H, Strehl U. Evaluation of neurofeedback in ADHD: the long and winding road. Biol Psychol 2014;95:108-115.

20. Higgins JP, Green S, editors. Cochrane Handbook for Systematic Reviews of Interventions 2011, John Wiley and Sons, Ltd, Chichester.

21. RevMan, version 5.1. Copenhagen, Nordic Cochrane Centre, Cochrane Collaboration, 2011

22. Morris SB. Estimating effect sizes from pretest-posttest-control group designs. Organ Res Methods. 2008;11:364-386.

23. StataCorp. 2013. Stata Statistical Software: Release 13. College Station, TX: StataCorp LP.

24. Linden M, Habib T, Radojevic V: A controlled study of the effects of EEG biofeedback on cognition and behavior of children with attention deficit disorder and learning disabilities. Biofeedback Self Regul 1996; 21:35-49.

25. Holtmann M, Grasmann D, Cionek-Szpak E et al. Specific effects of neurofeedback on impulsivity in ADHD. Kindheit und Entwicklung 2009; 18:95-104.

26. Arnold LE, Lofthouse N, Hersch S et al. EEG neurofeedback for ADHD: double-blind sham-controlled randomized pilot feasibility trial. J Atten Disord 2013;17(5):410-419.

27. Bakhshayesh AR, Hansch S, Wyschkon A, Rezai MJ, Esser G. Neurofeedback in ADHD: a single-blind randomized controlled trial. Eur Child Adolesc Psychiatry 2011;20(9):481-491.

28. Beauregard M, Levesque J. Functional magnetic resonance imaging investigation of the effects of neurofeedback training on the neural bases of selective attention and response 
inhibition in children with attention-deficit/hyperactivity disorder. Appl Psychophysiol Biofeedback 2006;31(1):3-20.

29. Bink M, van NC, Popma A, Bongers IL, van Boxtel GJ. Behavioral effects of neurofeedback in adolescents with ADHD: a randomized controlled trial. Eur Child Adolesc Psychiatry 2015;24(9):1035-1048.

30. Christiansen H, Reh V, Schmidt MH, Rief W. Slow cortical potential neurofeedback and self-management training in outpatient care for children with ADHD: study protocol and first preliminary results of a randomized controlled trial. Front Hum Neurosci 2014;8:943.

31. Gevensleben H, Holl B, Albrecht B et al. Is neurofeedback an efficacious treatment for ADHD? A randomised controlled clinical trial. J Child Psychol Psychiatry 2009;50(7):780789.

32. Heinrich H, Gevensleben H, Freisleder FJ, Moll GH, Rothenberger A. Training of slow cortical potentials in attention-deficit/hyperactivity disorder: evidence for positive behavioral and neurophysiological effects. Biol Psychiatry 2004;55(7):772-775.

33. Maurizio S, Liechti MD, Heinrich $\mathrm{H}$ et al. Comparing tomographic EEG neurofeedback and EMG biofeedback in children with attention-deficit/hyperactivity disorder. Biol Psychol 2014;95:31-44.

34. Steiner NJ, Sheldrick RC, Gotthelf D, Perrin EC. Computer-based attention training in the schools for children with attention deficit/hyperactivity disorder: a preliminary trial. Clin Pediatr (Phila) 2011;50(7):615-622.

35. Steiner NJ, Frenette EC, Rene KM, Brennan RT, Perrin EC. Neurofeedback and cognitive attention training for children with attention-deficit hyperactivity disorder in schools. J Dev Behav Pediatr 2014;35(1):18-27.

36. Vollebregt MA, van Dongen-Boomsma M, Buitelaar JK, Slaats-Willemse D. Does EEGneurofeedback improve neurocognitive functioning in children with attention- 
deficit/hyperactivity disorder? A systematic review and a double-blind placebo-controlled study. J Child Psychol Psychiatry 2014;55(5):460-472.

37. Gioia GA. BRIEF: Behavior rating inventory of executive function: Professional manual. Odessa: Psychological Assessment Resources; 2000.

38. Liechti MD, Valko L, Muller UC et al. Diagnostic value of resting electroencephalogram in attention-deficit/hyperactivity disorder across the lifespan. Brain Topogr 2013;26(1):135151.

39. Monastra VJ, Lubar JF, Linden M et al. Assessing attention deficit hyperactivity disorder via quantitative electroencephalography: an initial validation study. Neuropsychology 1999;13(3):424-433.

40. Heinrich H, Busch K, Studer P, Erbe K, Moll GH, Kratz O. EEG spectral analysis of attention in ADHD: implications for neurofeedback training? Front Hum Neurosci 2014;8:611.

41. Zuberer A, Brandeis D, Drechsler R. Are treatment effects of neurofeedback training in children with ADHD related to the successful regulation of brain activity? A review on the learning of regulation of brain activity and a contribution to the discussion on specificity. Front Hum Neurosci 2015;9:135.

42. Vernon D, Frick A, Gruzelier J. Neurofeedback as a treatment for ADHD: a methodological review with implications for future research. J Neurother 2004;8(2), 53-82.

43. Steiner NJ, Frenette EC, Rene KM, Brennan RT, Perrin EC. In-School Neurofeedback Training for ADHD: Sustained Improvements From a Randomized Control Trial. Pediatrics 2014;133(3):483-492.

44. Bink M, van NC, Popma A, Bongers IL, van Boxtel GJ. Neurocognitive effects of neurofeedback in adolescents with ADHD: a randomized controlled trial. J Clin Psychiatry 2014;75(5):535-542. 
45. van Dongen-Boomsma M, Vollebregt MA, Slaats-Willemse D, Buitelaar JK. A randomized placebo-controlled trial of electroencephalographic (EEG) neurofeedback in children with attention-deficit/hyperactivity disorder. J Clin Psychiatry 2013;74(8):821-827.

46. Wangler S, Gevensleben $\mathrm{H}$, Albrecht B et al. Neurofeedback in children with ADHD: specific event-related potential findings of a randomized controlled trial. Clin Neurophysiol 2011;122(5):942-950.

47. Arns M, Strehel U. Evidence for efficacy of neurofeedback in ADHD? Am J Psychiatry 2013;170(7):799-800.

48. Sonuga-Barke E, Brandeis D, Cortese S et al. Response to Chronis-Tuscano et al. and Arns and Strehel. Am J Psychiatry 2013;170(7):800-802. 
Table 1. Characteristics of studies included in the meta-analysis. Studies are listed in alphabetical order.

\begin{tabular}{|c|c|c|c|c|c|c|c|c|c|c|c|c|}
\hline \multirow[t]{3}{*}{ TRIAL A } & \multirow{3}{*}{$\begin{array}{c}\text { DESIGN } \\
\text { Control }\end{array}$} & \multicolumn{4}{|c|}{ TRAINING } & \multicolumn{3}{|c|}{ SAMPLE } & \multicolumn{4}{|c|}{ OUTCOMES } \\
\hline & & \multirow{2}{*}{$\begin{array}{l}\text { Type } \\
\text { NF }\end{array}$} & \multirow{2}{*}{$\begin{array}{l}\text { Standard } \\
\text { Protocol }\end{array}$} & \multirow{2}{*}{$\begin{array}{c}\mathbf{N} \\
\text { sessions }\end{array}$} & \multirow{2}{*}{$\begin{array}{c}\text { Follow- } \\
\text { up } \\
\text { (months) }\end{array}$} & \multirow{2}{*}{$\begin{array}{c}\mathbf{N}^{\mathrm{C}} \\
\mathbf{t} \\
\mathbf{c}\end{array}$} & \multirow{2}{*}{$\begin{array}{c}\text { meds }(\%) \\
\mathbf{t} \\
\mathrm{c}\end{array}$} & \multirow{2}{*}{$\begin{array}{c}\text { age } \\
\text { (mo.) }\end{array}$} & \multicolumn{2}{|c|}{ ADHD symptoms } & \multicolumn{2}{|c|}{ Neuropsychological outcomes } \\
\hline & & & & & & & & & $\begin{array}{c}\text { M- } \\
\text { PROX }\end{array}$ & $\begin{array}{c}\text { P- } \\
\text { BLIND }\end{array}$ & $\begin{array}{c}\text { Inhibitory } \\
\text { control }\end{array}$ & Attention \\
\hline $\begin{array}{l}\text { Arnold } \\
(2012)^{26}\end{array}$ & $\begin{array}{c}\text { Sham } \\
\text { placebo }\end{array}$ & FTB $^{\mathbf{E}}$ & no & 40 & 2 & $\begin{array}{l}25 \\
11\end{array}$ & $\begin{array}{l}0 \\
0\end{array}$ & $72-144$ & Parent & Teacher & & BRC \\
\hline $\begin{array}{c}\text { Bakhshayesh } \\
\text { (2011) }^{\mathbf{2 7}}\end{array}$ & $\begin{array}{c}\text { EMG } \\
\text { biofeedback }\end{array}$ & FTB & yes & 30 & $6^{\mathbf{F}}$ & $\begin{array}{l}18 \\
17\end{array}$ & $\begin{array}{l}22 \\
18\end{array}$ & $72-168$ & Parent & Teacher & $\begin{array}{c}\text { CPT } \\
\text { (commission) }\end{array}$ & CPT (omission) \\
\hline $\begin{array}{c}\text { Beauregard } \\
(2006)^{\mathbf{2 8}}\end{array}$ & $\begin{array}{c}\text { No } \\
\text { treatment }\end{array}$ & FTB $^{\mathbf{~}}$ & yes & 40 & $\mathrm{~N} / \mathrm{S}$ & $\begin{array}{c}15 \\
5\end{array}$ & $\begin{array}{l}0 \\
0\end{array}$ & $96-144$ & Parent & & $\begin{array}{c}\text { Counting } \\
\text { Stroop } \\
\text { (interference) } \\
\end{array}$ & $\begin{array}{c}\text { CPT (integrated } \\
\text { visual and } \\
\text { auditory) } \\
\end{array}$ \\
\hline $\begin{array}{c}\text { Bink } \\
(2014)^{29}\end{array}$ & TAU & FTB $^{\mathbf{H}}$ & no & 37 & 12 & $\begin{array}{l}45 \\
26\end{array}$ & $\begin{array}{l}44.4 \\
61.5\end{array}$ & $\begin{array}{c}193.2 \\
\pm 39.6 \\
(\mathrm{~T}) \\
194.4 \pm \\
40.8(\mathrm{C}) \\
\end{array}$ & Parent & & $\begin{array}{l}\text { Colour Stroop } \\
\text { (Interference) }\end{array}$ & $\begin{array}{l}\text { D2 attention } \\
\text { (total correct) }\end{array}$ \\
\hline $\begin{array}{c}\text { Christiansen } \\
\text { (2014) }^{\mathbf{3 0}}\end{array}$ & Self-manag. & $\mathrm{SCP}$ & yes & 30 & 12 & $\begin{array}{c}58 \\
\text { (tot.) }\end{array}$ & $23^{\mathrm{I}, \mathrm{L}}$ & $\begin{array}{l}101.04 \\
\pm 16.08\end{array}$ & Parent & Teacher & & \\
\hline $\begin{array}{c}\text { Gevensleben } \\
\text { (2009) } \mathbf{3 1}\end{array}$ & AT & $\begin{array}{c}\text { SCP } \\
\text { plus } \\
\text { FTB }^{\text {M }}\end{array}$ & yes & 36 & 6 & $\begin{array}{l}59 \\
35\end{array}$ & $\begin{array}{l}8.5 \\
2.9\end{array}$ & $\begin{array}{c}118 \\
\pm 15(\mathrm{~T}) \\
112 \pm \\
14(\mathrm{C})\end{array}$ & Parent & Teacher & $\begin{array}{c}\text { ANT, } \\
\text { Orienting } \\
\text { (data from }{ }^{46}, \\
\text { that refers to } \\
\text { the same } \\
\text { sample of }{ }^{31} \text { ) }\end{array}$ & $\begin{array}{c}\text { ANT, } \\
\text { Conflict } \\
\text { (data from }{ }^{46}, \\
\text { that refers to the } \\
\text { same sample of } \\
{ }_{31} \text { ) }\end{array}$ \\
\hline
\end{tabular}




\begin{tabular}{|c|c|c|c|c|c|c|c|c|c|c|c|c|}
\hline $\begin{array}{l}\text { Heinrich } \\
(2004)^{32}\end{array}$ & WL & $\mathrm{SCP}^{\mathrm{N}}$ & yes & 25 & $\mathrm{~N} / \mathrm{S}$ & $\begin{array}{c}13 \\
9\end{array}$ & $\begin{array}{l}46.1 \\
44.4\end{array}$ & $90-165$ & Parent & & $\begin{array}{c}\mathrm{CPT} \\
\text { (commission) }\end{array}$ & CPT (omission) \\
\hline $\begin{array}{l}\text { Holtmann } \\
(2009)^{25}\end{array}$ & $\mathrm{AT}^{\mathrm{O}}$ & FTB $^{N}$ & yes & 20 & $\mathrm{~N} / \mathrm{S}$ & $\begin{array}{l}20 \\
14\end{array}$ & $79.4^{\mathbf{P}}$ & $\begin{array}{c}123.6 \pm \\
14.4 \\
\text { mean } \\
\end{array}$ & Parent & & $\begin{array}{c}\text { Stop Signal } \\
\text { Task }\end{array}$ & \\
\hline $\begin{array}{l}\text { Linden } \\
(1996)^{24}\end{array}$ & WL & FTB & yes & 40 & $\mathrm{~N} / \mathrm{S}$ & $\begin{array}{l}8 \\
6\end{array}$ & $\begin{array}{l}0 \\
0\end{array}$ & $\begin{array}{c}110 \\
(\text { mean })\end{array}$ & Parent & & & \\
\hline $\begin{array}{l}\text { Maurizio } \\
(2014)^{33}\end{array}$ & $\begin{array}{c}\text { EMG } \\
\text { biofeedback }\end{array}$ & $\begin{array}{c}\text { SCP } \\
\text { plus } \\
\text { FTB Q } \\
\end{array}$ & no & 36 & $\mathrm{~N} / \mathrm{S}$ & $\begin{array}{l}13 \\
12\end{array}$ & $\begin{array}{l}7.6^{\mathbf{I}} \\
8.3^{\mathbf{I}}\end{array}$ & $\begin{array}{l}102- \\
154.8\end{array}$ & Parent & Teacher & & $\begin{array}{l}\text { D2 attention } \\
\text { (total score) }\end{array}$ \\
\hline $\begin{array}{c}\text { Steiner } \\
(2011)^{\mathbf{3 4} \mathbf{R}}\end{array}$ & $\mathrm{AT}^{\mathbf{o}}, \mathrm{WL}^{\mathbf{s}}$ & FTB $^{\mathbf{H}}$ & no & 23 & $\mathrm{~N} / \mathrm{S}$ & $\begin{array}{c}9 \\
11\end{array}$ & 60 & $\begin{array}{c}148.8 \pm \\
10.8\end{array}$ & Parent & Teacher & & \\
\hline $\begin{array}{l}\text { Steiner } \\
(2014)^{35}\end{array}$ & $\mathrm{CT}, \mathrm{WL}^{\mathrm{s}}$ & FTB $^{\mathbf{H}}$ & no & 40 & $6^{T}$ & $\begin{array}{l}34 \\
34\end{array}$ & $\begin{array}{l}44.1 \\
41.1\end{array}$ & $\begin{array}{c}100.8 \pm \\
13.2 \\
(\mathrm{~T}) \\
106.8 \pm \\
12.0 \\
(\mathrm{C}) \\
\end{array}$ & Parent & BOSS & & \\
\hline $\begin{array}{l}\text { VanDongen } \\
(2013)^{\mathbf{4 5}} \\
\text { Vollebregt } \\
(2014)^{\mathbf{3 6}, \mathbf{U}}\end{array}$ & Placebo NF & FTB v & no & 30 & $6^{z}$ & $\begin{array}{l}22 \\
19\end{array}$ & $\begin{array}{l}54.5 \\
73.7\end{array}$ & $\begin{array}{c}126.0 \pm \\
26.4 \\
(\mathrm{~T}) \\
128.4 \pm \\
27.6(\mathrm{C}\end{array}$ & $\begin{array}{l}\text { Investi } \\
\text { gator }\end{array}$ & Teacher & & \\
\hline
\end{tabular}


NOTES:

ANT: Attention Network Test; AT: attention training; BOSS: Behavioral Observation of Students in Schools; BRC: brain resource center computer based normed neuropsychological test; C: Control Group; CPT: continuous performance test; CT: cognitive training; EMG: electromyography; FTB; frequency theta/beta; NF: neurofeedback; SCP: Slow Cortical Potential; SMR: sensory motor rhythm; SST: Stop Signal Test; TAU: Treatment As Usual; WL: waiting list

${ }^{\mathbf{A}}$ In alphabetic order, followed by study reference number; ${ }^{\mathbf{B}}$ Standard methodology described in ${ }^{19} ; \mathbf{C}^{\mathbf{C}} \mathrm{N}$ is the number of individuals in the Treatment (T) and Control (C) conditions; ${ }^{\mathbf{D}}$ Equal intensity and duration; ${ }^{\mathbf{E}} \mathbf{C z}$ vs ears; ${ }^{\mathbf{F}}$ Results of the follow-up were not published in this paper; ${ }^{\mathbf{G}}$ SMR+beta; ; ${ }^{\text {SMR; }}{ }^{\mathbf{I}}$ Children were off medication $48 \mathrm{~h}$ prior to all the assessments; ${ }^{\mathbf{L}}$ Number in each group not specified; ${ }^{\mathbf{M}} \mathrm{SMR}+\mathrm{beta} \mathrm{Cz}-\mathrm{ears}$; ${ }^{\mathbf{N}}$ Calculated from $\mathrm{Cz}$ vs mastoids; ${ }^{\mathbf{O}}$ standard computer format; ${ }^{\mathbf{P}}$ A total of $\mathrm{n}=27$ were medicated (not indicated how many in each group); $\mathbf{Q}$

$\mathrm{B}=$ beta, LORETA Tomography; ${ }^{\mathbf{R}}$ We used Parent\#1 since parent \#2 measures were available for a smaller sample ( $\mathrm{n}=9$ for parent \#1 and $\mathrm{n}=5$ for parent \#2 in NF and $n=11$ for parent \#1 in $n=9$ for parent \#2 in the waiting list group); for a discussion of the outcomes selected from this study, please see ${ }^{47}$ and ${ }^{48}$; $\mathbf{S}$ : The WL arm was used as comparator in the analyses; ${ }^{\mathbf{T}}$ The results of the follow-up phase were published in ${ }^{43}$; ${ }^{\mathbf{U}}$ These two papers refers to the same study and present analyses on different outcomes; ${ }^{\mathbf{V}}$ Individualized, mainly $\mathrm{T} / \mathrm{B}, \mathrm{B}=\mathrm{SMR} ;{ }^{\mathbf{Z}}$ data only reported at endpoint (15 weeks) 
Table 2. Summary of results. Pooled standardized mean differences (SMD) for each outcomes.

Positive SMDs indicate that neurofeedback is more efficacious than control condition.

\begin{tabular}{|c|c|c|c|c|c|c|c|c|}
\hline \multirow[t]{2}{*}{ Outcome } & \multirow{2}{*}{$\begin{array}{c}\text { Trials } \\
\text { included }\end{array}$} & \multirow[t]{2}{*}{ Measure } & \multirow{2}{*}{$\begin{array}{l}\text { Number } \\
\text { of trials }\end{array}$} & \multicolumn{3}{|c|}{ Effect of Intervention } & \multicolumn{2}{|c|}{ Heterogeneity } \\
\hline & & & & SMD & $95 \% \mathrm{CI}$ & $\mathbf{p}$ & $\mathbf{I}^{2}$ & $\mathbf{p}$ \\
\hline \multicolumn{9}{|c|}{ ADHD symptoms } \\
\hline \multirow{9}{*}{ Total } & \multirow{2}{*}{ All } & MPROX & 13 & 0.35 & $0.11,0.59$ & 0.004 & 41 & 0.06 \\
\hline & & PBLIND & 8 & 0.15 & $-0.08,0.38$ & 0.20 & 0 & 0.74 \\
\hline & \multirow{2}{*}{ Active/sham } & MPROX & 7 & 0.22 & $-0.08,0.52$ & 0.14 & 35 & 0.16 \\
\hline & & PBLIND & 6 & 0.20 & $-0.05,0.45$ & 0.12 & 0 & 0.66 \\
\hline & \multirow{2}{*}{ FBT } & MPROX & 9 & 0.37 & $0.09,0.64$ & 0.01 & 36 & 0.13 \\
\hline & & PBLIND & 5 & 0.03 & $-0.29,0.35$ & 0.84 & 0 & 0.76 \\
\hline & \multirow{2}{*}{$\begin{array}{c}\text { Low } \\
\text { medication }\end{array}$} & MPROX & 7 & 0.39 & $-0.01,0.79$ & 0.05 & 48 & 0.08 \\
\hline & & PBLIND & 5 & 0.26 & $-0.01,0.54$ & 0.06 & 0 & 0.76 \\
\hline & $\begin{array}{l}\text { Standard } \\
\text { Protocol }\end{array}$ & MPROX & 7 & 0.45 & $0.02,0.88$ & 0.04 & 53 & 0.05 \\
\hline \multirow{8}{*}{ inattention } & \multirow{2}{*}{ All } & MPROX & 11 & 0.36 & $0.09,0.63$ & 0.009 & 43 & 0.07 \\
\hline & & PBLIND & 7 & 0.06 & $-0.24,0.36$ & 0.70 & 41 & 0.12 \\
\hline & \multirow{2}{*}{ Active/sham } & MPROX & 6 & 0.26 & $-0.10,0.63$ & 0.16 & 49 & 0.08 \\
\hline & & PBLIND & 5 & 0.21 & $-0.09,0.50$ & 0.17 & 16 & 0.31 \\
\hline & \multirow{2}{*}{ FBT } & MPROX & 9 & 0.33 & $0.00,0.67$ & 0.05 & 51 & 0.04 \\
\hline & & PBLIND & 5 & -0.04 & $-0.37,0.28$ & 0.79 & 19 & 0.29 \\
\hline & $\begin{array}{c}\text { Low } \\
\text { medication }\end{array}$ & MPROX & 6 & 0.59 & $0.31,0.88$ & 0.0001 & 0 & 0.63 \\
\hline & $\begin{array}{l}\text { Standard } \\
\text { Protocol }\end{array}$ & MPROX & 5 & 0.55 & $0.01,1.09$ & 0.05 & 58 & 0.05 \\
\hline
\end{tabular}




\begin{tabular}{|c|c|c|c|c|c|c|c|c|}
\hline \multirow{7}{*}{ hyper/imp } & \multirow{2}{*}{ All } & MPROX & 10 & 0.26 & $0.08,0.43$ & 0.004 & 0 & 0.80 \\
\hline & & PBLIND & 7 & 0.17 & $-0.05,0.39$ & 0.13 & 0 & 0.59 \\
\hline & \multirow{2}{*}{ Active/sham } & MPROX & 6 & 0.25 & $0.03,0.47$ & 0.03 & 0 & 0.92 \\
\hline & & PBLIND & 5 & 0.15 & $-0.11,0.41$ & 0.26 & 0 & 0.80 \\
\hline & \multirow{2}{*}{ FBT } & MPROX & 8 & 0.26 & $0.06,0.46$ & 0.01 & 0 & 0.62 \\
\hline & & PBLIND & 5 & 0.15 & $-0.15,0.44$ & 0.33 & 6 & 0.37 \\
\hline & $\begin{array}{c}\text { Low } \\
\text { medication }\end{array}$ & MPROX & 5 & 0.32 & $0.06,0.58$ & 0.02 & 0 & 0.44 \\
\hline \multicolumn{9}{|c|}{ Neuropsychological Test Performance } \\
\hline \multirow{2}{*}{ attention } & All & objective & 8 & 0.13 & $-0.09,0.36$ & 0.26 & 0 & 0.72 \\
\hline & FBT & objective & 5 & 0.11 & $-0.16,0.38$ & 0.43 & 0 & 0.76 \\
\hline inhibition & All & objective & 6 & 0.30 & $-0.10,0.70$ & 0.15 & 56 & 0.05 \\
\hline
\end{tabular}

\section{NOTES:}

\section{Significant effects are bolded}

There were insufficient $(\mathrm{n}<5)$ trials for: standard NF, PBLIND tot $(\mathrm{n}=3)$, PBLIND In $(\mathrm{n}=3)$; Standard protocol, MPROX, hyp/imp ( $n=4)$ and PBLIND (tot, In, hyper/imp) $(n=3) ;$ FBT, inhibition ( $n=4$ trials); low medication, Inattention, PBLIND $(n=4)$ and Hyp/imp, PBLIND, $(n=4)$. "All" = all trials meeting inclusion criteria with available measures; "active/sham" = only trials with an active/sham control arm; CI: confidence interval; FBT = trials using a version of frequency band training including theta-beta ratio training. MPROX: most proximal rater; PBLIND: probably blinded rater; SMT: Self-management Therapy; TAU: treatment as usual 


\section{FIGURES TITLES}

Figure 1. PRISMA flow diagram of selection of studies (last search updated on August $30^{\text {th }}$, 2015).

NOTE $^{\mathrm{A}}$ Reasons for exclusion of each paper are reported in Table S1 ; ${ }^{\mathrm{B}}$ Four papers in the search $\left({ }^{29},{ }^{35},{ }^{36},{ }^{46}\right)$ refer to the same sample of other $4\left({ }^{44},{ }^{43},{ }^{45},{ }^{31}\right.$, respectively)

Figure 2. Forest plots for meta-analysis of effects on ADHD core symptoms assessed by most proximal (MPROX) raters.

NOTE a: ADHD total symptoms; b: inattentive symptoms; c: hyperactive/impulsive symptoms SMD: standardized mean difference

Figure 3. Forest plots for meta-analysis of effects on ADHD core symptoms assessed by probably blinded (PBLIND) raters.

NOTE a: ADHD total symptoms; b: inattentive symptoms; c: hyperactive/impulsive symptoms SMD: standardized mean difference 


\section{SUPPLEMENTAL FIGURES TITLES}

Figure S1. Cochrane risk of bias tool: Graphic output

Figure S2 . Cochrane risk of bias tool: characteristic of included studies

Figure S3. Funnel Plots and Egger's Tests for Meta-Analyses of Effects of Neurofeedback on Attention-Deficit/Hyperactivity Disorder (ADHD) Core Symptoms (Most Proximal and Probably Blinded Measures): Most Proximal Measures (MPROX)

Figure S4. Funnel Plots and Egger's Tests for Meta-Analyses of Effects of Neurofeedback on Attention-Deficit/Hyperactivity Disorder (ADHD) Core Symptoms (Most Proximal and Probably Blinded Measures): Probably blinded measures (PBLIND)

Figure S5. Results of the Meta-Regression Analysis to Assess the Relationship Between Number of Neurofeedback sessions and Standardized Mean Difference (SMD) for Most Proximal and Probably Blinded Assessment of Attention-Deficit/Hyperactivity Disorder (ADHD) Core Symptoms: Most Proximal Measures (MPROX)

Figure S6. Results of the Meta-Regression Analysis to Assess the Relationship Between Number of Neurofeedback sessions and Standardized Mean Difference (SMD) for Most Proximal and Probably Blinded Assessment of Attention-Deficit/Hyperactivity Disorder (ADHD) Core Symptoms: Probably Blinded Measures (PBLIND) 